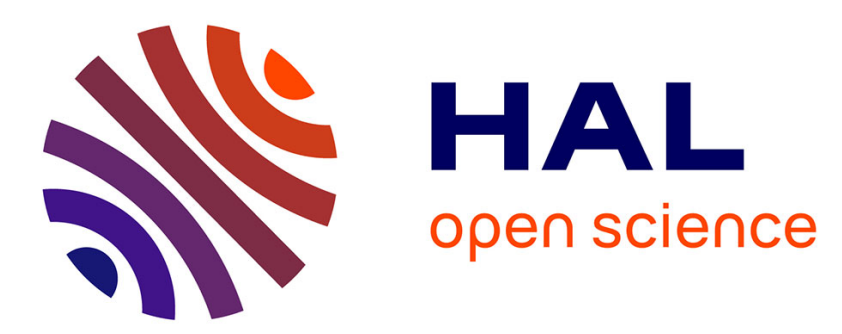

\title{
Dérivabilité ponctuelle d'une intégrale liée aux fonctions de Bernoulli
}

Régis de La Bretèche, Gérald Tenenbaum

\section{To cite this version:}

Régis de La Bretèche, Gérald Tenenbaum. Dérivabilité ponctuelle d'une intégrale liée aux fonctions de Bernoulli. Proceedings of the American Mathematical Society, 2015, 143 (11), pp.4791-4796. 10.1090/S0002-9939-2015-12650-0 . hal-01281584

\section{HAL Id: hal-01281584 \\ https://hal.science/hal-01281584}

Submitted on 2 Mar 2016

HAL is a multi-disciplinary open access archive for the deposit and dissemination of scientific research documents, whether they are published or not. The documents may come from teaching and research institutions in France or abroad, or from public or private research centers.
L'archive ouverte pluridisciplinaire HAL, est destinée au dépôt et à la diffusion de documents scientifiques de niveau recherche, publiés ou non, émanant des établissements d'enseignement et de recherche français ou étrangers, des laboratoires publics ou privés. 


\title{
Dérivabilité ponctuelle d'une intégrale liée aux fonctions de Bernoulli
}

\author{
R. de la Bretèche \& G. Tenenbaum
}

\begin{abstract}
Let $B(\vartheta)$ denote the first normalised Bernoulli function, and consider the series $f(\vartheta):=\sum_{n \geqslant 1} B(n \vartheta) / n$. In a previous paper [3], we determined the set $E^{*}$ of those real numbers $\vartheta$ at which $f(\vartheta)$ converges. Let $B_{2}(\vartheta)$ designate the second Bernoulli function and $\langle t\rangle$ denote the fractional part of the real number $t$. Put $F(\vartheta):=\sum_{n \geqslant 1} B_{2}(n \vartheta) / 2 n^{2}=\pi^{2} / 72+\int_{0}^{\vartheta} f(t) \mathrm{d} t$. It has been shown by Báez-Duarte, Balazard, Landreau and Saias [1] that $F(\vartheta)$ and $\int_{0}^{\infty}\langle t\rangle\langle\vartheta t\rangle \mathrm{d} t / t^{2}$ have the same differentiability points. Moreover, using delicate functional equations, Balazard and Martin [2] recently proved that the corresponding set is precisely $E:=E^{*} \backslash \mathbb{Q}$ and that $F^{\prime}(\vartheta)=f(\vartheta)$ whenever $\vartheta \in E$. We provide a short, direct proof of this last result, based on standard results from Diophantine approximation theory and uniform distribution theory.

Keywords: Bernoulli functions, differentiability, Diophantine approximation, continued fractions, distribution modulo one, Erdős-Turán inequality, Davenport identities.
\end{abstract}

L'un des problèmes les plus fondamentaux et les plus délicats de l'analyse mathématique consiste à déterminer sous quelles conditions l'intégration et la différentiation sont des opérations réciproques - voir par exemple le chapitre XI du livre de Titchmarsh [8]. Spécifiquement considérons une fonction $f$ Lebesgue-intégrable sur un sous-intervalle $I$ de $\mathbb{R}$ contenant un point $a$ et son intégrale indéfinie

$$
F(x):=\int_{a}^{x} f(t) \mathrm{d} t \quad(x \in I) .
$$

Selon un théorème de Lebesgue (cf., par exemple, [8], §11.6, ou [6], th.7.11) presque tout élement $x$ de $I$ est un point de Lebesgue de $f$; en un tel point, la fonction $F$ est dérivable et vérifie

$$
F^{\prime}(x)=f(x)
$$

Cependant, la description précise de l'ensemble des points $x$ où la relation (1) est satisfaite demeure en général un problème difficile.

Selon un résultat classique (voir par exemple le théorème 4.10.7 de Saks [7]), la relation (1) a lieu dès que $f$ est bornée au voisinage de $x$ si $x$ est un point de continuité approximative pour $f$, autrement dit satisfaisant à la condition

$$
(\forall \eta>0) \quad \liminf _{\varepsilon \rightarrow 0} \operatorname{mes}\{t \in[x-\varepsilon, x+\varepsilon]:|f(t)-f(x)|<\eta\} /(2 \varepsilon)=1 .
$$

Cependant, comme l'a montré Sarkhel [9] et contrairement à une assertion de Munroe [4], la continuité approximative n'est pas nécessaire à la validité de (1).

Lorsque $f$ n'est pas bornée, la situation est beaucoup plus délicate encore. Sarkhel donne, pour les points de continuité approximative, une condition nécessaire et une condition suffisante qui sont peu exploitables en pratique.

Nous considérons ici le cas particulier d'une fonction $f$ non bornée définie à l'aide des fonctions de Bernoulli. Cette étude spécifique apparaît en fait comme emblématique d'une classe plus large de problèmes dans lesquels les approximations rationnelles de la variable jouent un rôle prédominant dans l'approximation de la fonction.

Soit $B(\vartheta)$ la première fonction de Bernoulli normalisée, i.e.

$$
B(\vartheta):= \begin{cases}\langle\vartheta\rangle-\frac{1}{2} & \text { si } \vartheta \notin \mathbb{Z} \\ 0 & \text { si } \vartheta \in \mathbb{Z}\end{cases}
$$

2010 Mathematics Subject Classification: primary 26A24, 26A27; secondary 11J70, 11J71, 11K06, 11L07, 11M26. 
où $\langle\vartheta\rangle$ désigne la partie fractionnaire du nombre réel $\vartheta$. Posons alors

$$
f(\vartheta):=\sum_{n \geqslant 1} \frac{B(n \vartheta)}{n}
$$

lorsque la série converge et, par exemple, $f(\vartheta):=0$ dans le cas contraire. Introduisons encore l'intégrale indéfinie

$$
F(\vartheta):=\sum_{n \geqslant 1} \frac{B_{2}(n \vartheta)}{2 n^{2}}=\frac{1}{72} \pi^{2}+\int_{0}^{\vartheta} f(t) \mathrm{d} t,
$$

où $B_{2}(\vartheta):=\langle\vartheta\rangle^{2}-\langle\vartheta\rangle+\frac{1}{6}=2 \int_{0}^{\vartheta} B(t) \mathrm{d} t+\frac{1}{6}$ est la deuxième fonction de Bernoulli. ${ }^{(1)}$

Soit $E$ l'ensemble des nombres réels irrationnels $\vartheta$ tels que la série

$$
\sum_{j \geqslant 0}(-1)^{j} \frac{\log q_{j+1}}{q_{j}}
$$

converge, où $\left\{q_{j}\right\}_{j=0}^{\infty}$ est la suite des dénominateurs des réduites de $\vartheta$. Il est établi dans [3] que la série (2) converge si, et seulement si, $\vartheta \in E \cup \mathbb{Q}$.

Il n'est pas difficile de montrer grâce aux estimations de [3] que $f$ est approximativement continue sur $E$, mais, comme $f$ n'est pas bornée, ${ }^{(2)}$ on ne peut en déduire directement que $E$ coïncide avec l'ensemble des points de dérivabilité de $F(\vartheta)$.

Ce problème a été étudié et résolu par Balazard et Martin dans le travail [2], dévolu à l'étude de la fonction d'auto-corrélation multiplicative

$$
A(\vartheta):=\int_{0}^{\infty}\langle t\rangle\langle\vartheta t\rangle \frac{\mathrm{d} t}{t^{2}} \quad(\vartheta>0),
$$

introduite dans le travail [1] dans le cadre de l'étude du critère de Nyman pour l'hypothèse de Riemann. Le lien entre les deux problèmes est immédiat au vu de la formule établie dans [1]

$$
A(\vartheta)=\frac{1}{2} \log \vartheta+\frac{1}{2} A(1)+\frac{1}{2}-\vartheta \int_{\vartheta}^{\infty} \frac{f(t)}{t^{2}} \mathrm{~d} t \quad(\vartheta>0) .
$$

Les points de dérivabilité de $A$ et $F$ sont donc les mêmes.

En suivant une méthode basée sur de délicates exploitations d'équations fonctionnelles, Balazard et Martin établissent dans [2] le résultat suivant.

Théorème 1. La fonction $F$ est dérivable en $\vartheta$ si, et seulement si, $\vartheta \in E$, auquel cas on a $F^{\prime}(\vartheta)=f(\vartheta)$.

La présente note a pour objet de proposer une nouvelle démonstration de ce résultat par une méthode directe, indépendante de celle de [2], et reposant simplement sur les estimations de $f(\vartheta)$ obtenues dans [3].

Démonstration. Posons

$$
f_{N}(t):=\sum_{1 \leqslant n \leqslant N} \frac{B(n t)}{n} \quad(t \in \mathbb{R}, N \geqslant 1),
$$

de sorte que, pour tout entier $N \geqslant 1$, tous $\vartheta \in \mathbb{R}, h \in \mathbb{R}^{*}$, nous pouvons écrire

$$
\frac{F(\vartheta+h)-F(\vartheta)}{h}=\frac{1}{h} \int_{\vartheta}^{\vartheta+h} f_{N}(t) \mathrm{d} t+\sum_{n>N} \frac{B_{2}(n \vartheta+n h)-B_{2}(n \vartheta)}{2 n^{2} h} .
$$

1. L'intégration terme à terme est justifiée par le fait que la série $f$ converge dans $L^{2}[0,1]$ puisque $\int_{0}^{1} B(\ell \vartheta) B(n \vartheta) \mathrm{d} \vartheta=(\ell, n) /[\ell, n]$ pour tous entiers $\ell \geqslant 1, n \geqslant 1$.

2. Voir (11) et (12) infra. 
Considérons alors un nombre réel $\vartheta$ et désignons par $\left\{p_{j} / q_{j}\right\}_{j \geqslant 0}$ la suite finie ou infinie de ses réduites. Rappelons que, si $\vartheta \neq p_{j} / q_{j}$,

$$
1 / 2 q_{j+1}<\left\|q_{j} \vartheta\right\| \leqslant 1 / q_{j+1} \quad(j \geqslant 1)
$$

où $\|\cdot\|$ désigne la distance à l'ensemble des entiers. Soit $R \geqslant 1$. Si $|t-\vartheta| \leqslant 1 / q_{j} q_{j+1}$ et $n<\frac{1}{2} R q_{j+1}$, nous avons $B(n t)-B(n \vartheta)=n(t-\vartheta)$ sauf peut-être lorsqu'il existe un entier $v \in[-R, R]$ tel que $n p_{j} \equiv v\left(\bmod q_{j}\right)$ et $\frac{1}{2} q_{j+1} \leqslant n<\frac{1}{2} R q_{j+1}$. En effet, nous déduisons des inégalités

$$
\left|n t-n p_{j} / q_{j}\right| \leqslant\left|n \vartheta-n p_{j} / q_{j}\right|+n / q_{j} q_{j+1} \leqslant 2 n / q_{j+1}<R / q_{j}
$$

que, si $v \in\left[-\frac{1}{2} q_{j}, \frac{1}{2} q_{j}\right]$ est tel que $n p_{j} \equiv v\left(\bmod q_{j}\right)$, alors $\lfloor n t\rfloor=\lfloor n \vartheta\rfloor$ dès que $|v|>R$ et donc $B(n t)-B(n \vartheta)=n(t-\vartheta)$. Ainsi, pour $j \geqslant 1, x \geqslant 1$, et en choisissant $R:=1+2 x / q_{j+1}$, nous pouvons écrire

$$
\sum_{n \leqslant x}\{B(n t)-B(n \vartheta)\} \ll \frac{R x}{q_{j}}+\sum_{n \leqslant x} n|t-\vartheta| \ll \frac{x}{q_{j}}+\frac{x^{2}}{q_{j} q_{j+1}} \quad\left(|\vartheta-t| \leqslant 1 / q_{j} q_{j+1}\right) .
$$

Notons $\left[\xi_{j}, \xi_{j+1}\right.$ [ l'intervalle constitué des nombres réels $x>1$ satisfaisant aux inégalités $q_{j} \leqslant Q_{x}<q_{j+1}$, pour le choix $Q_{x}:=x /(\log x)^{30}$. Nous avons alors, en vertu du lemme 11.3 de [3], pour $\xi_{j} \leqslant x<\xi_{j+1}$,

$$
W(x ; \vartheta):=\sum_{n \leqslant x} B(n \vartheta) \ll \begin{cases}\frac{x}{q_{j}}+\frac{x}{(\log x)^{2}} & \text { si } \xi_{j} \leqslant x \leqslant \max \left(\xi_{j}, q_{j+1}\right), \\ \frac{q_{j+1}}{q_{j}}+\frac{x}{(\log x)^{2}} & \text { si } \max \left(\xi_{j}, q_{j+1}\right)<x<\xi_{j+1} .\end{cases}
$$

De plus, un examen de la preuve permet de montrer que cette majoration est également valable en remplaçant $\vartheta$ par $t$ sous réserve que $|t-\vartheta|<1 / 3 q_{j} q_{j+1}$, ce qui implique $1 / 6 \leqslant\left\|q_{j} t\right\| q_{j+1} \leqslant 2$.

Nous aurons également besoin d'une estimation uniforme pour la somme $W(x ; t)$. À cette fin nous faisons appel à l'inégalité d'Erdős-Turán (voir par exemple [5] pour les références historiques et la version actuellement la plus précise) sous la forme

$$
|W(x ; t)| \leqslant x D_{x}(t) \leqslant \frac{x}{K}+\sum_{1 \leqslant k \leqslant K} \frac{1}{k} \min \left(x, \frac{1}{\|k t\|}\right) \quad(K \geqslant 1)
$$

où $D_{x}(t)$ désigne la discrépance de la suite $\{\langle n t\rangle\}_{n \leqslant x}$. Si $K:=q / 3,|t-a / q| \leqslant 2 / q^{2}, 1 \leqslant k \leqslant K$, l'argument employé pour établir le lemme des six valeurs (voir [3], lemme 6.3) implique que, pour chaque entier $r$ de $[1, q / 2]$, il existe au plus six valeurs de $k$ telles que $\|k t\| \in] r / q,(r+1) / q]$. Désignant par $k_{r}$ le plus petit de ces entiers, nous avons donc

$$
\sum_{1 \leqslant k \leqslant q / 3} \frac{1}{k\|k t\|} \leqslant 6 \sum_{\substack{1 \leqslant r \leqslant q / 2 \\ k_{r} \leqslant q / 3}} \frac{q}{r k_{r}} .
$$

En vertu d'une inégalité de réarrangement classique, cette dernière somme est maximale lorsque la suite $k_{r}$ est croissante. Comme l'application $r \mapsto k_{r}$ est injective, il suit

$$
\sum_{1 \leqslant r \leqslant q / 2} \frac{1}{r k_{r}} \leqslant \sum_{r \geqslant 1} \frac{1}{r^{2}}=\frac{1}{6} \pi^{2}
$$

Nous obtenons ainsi, pour tous entiers $a, q$, tels que $(a, q)=1$,

$$
W(x ; t) \leqslant x D_{x}(t) \ll \frac{x}{q}+q \quad\left(x \geqslant 1,|t-a / q| \leqslant 2 / q^{2}\right) .
$$


Soit $m \geqslant 1$. Employons (5), (6) et (8) pour estimer $f_{N}(t)-f_{N}(\vartheta)$ par sommation d'Abel lorsque $h>0,|t-\vartheta| \leqslant h$, et

$$
3 q_{m}^{2} \leqslant 1 / h<3 q_{m+1}^{2}, \quad N:=\lfloor 1 / h\rfloor .
$$

Choisissant alors $j=m-1$ dans (5) et (6), et $q=q_{m}$ dans (8), nous obtenons, pour $|t-\vartheta| \leqslant h$,

$$
\begin{aligned}
f_{N}(t)-f_{N}(\vartheta) & \ll \int_{1}^{\max \left(\xi_{m-1}, q_{m}\right)}\left\{\frac{1}{x q_{m-1}}+\frac{1}{q_{m-1} q_{m}}\right\} \mathrm{d} x \\
& +\int_{\max \left(\xi_{m-1}, q_{m}\right)}^{\xi_{m}}\left\{\frac{q_{m}}{q_{m-1} x^{2}}+\frac{1}{x(\log x)^{2}}\right\} \mathrm{d} x+\int_{\xi_{m}}^{N}\left\{\frac{1}{x q_{m}}+\frac{q_{m}}{x^{2}}\right\} \mathrm{d} x \\
& \ll \frac{\log q_{m}}{q_{m-1}}+\frac{1}{\log q_{m}}+\frac{\log q_{m+1}}{q_{m}} .
\end{aligned}
$$

Par ailleurs, puisque $B_{2}$ est continue et dérivable par morceaux, la majoration (7), et donc (8), est également valide en remplaçant la fonction $B$ par la fonction $B_{2}$ dans la définition de $W(x, t)$. Il s'ensuit que, pour $x>3 q_{m}^{2}$, nous avons

$$
\sum_{n \leqslant x} B_{2}(n t) \ll \frac{x}{q_{m}} .
$$

Par sommation d'Abel, cela implique

$$
\sum_{n>N} \frac{B_{2}(n \vartheta \pm n h)-B_{2}(n \vartheta)}{2 n^{2} h} \ll \frac{1}{N h q_{m}} \ll \frac{1}{q_{m}}
$$

En reportant dans (4) et en faisant tendre $m$ vers l'infini, nous obtenons, donc, pour tout $\vartheta \in E$,

$$
\frac{F(\vartheta \pm h)-F(\vartheta)}{ \pm h}=f_{N}(\vartheta)+o(1)
$$

Cela montre bien que $F$ est dérivable en $\vartheta$ et vérifie $F^{\prime}(\vartheta)=f(\vartheta)$.

Il reste à traiter le cas $\vartheta \in \mathbb{R} \backslash E$.

Lorsque $\vartheta \in \mathbb{Q}$, les auteurs de [1] ont établi que $F(\vartheta+h)-F(\vartheta) \asymp h \log (1 /|h|) \operatorname{lorsque}$ $h \rightarrow 0$. Nous pouvons obtenir simplement la non-dérivabilité de $F$ en observant que, pour $\vartheta:=p / q, N:=\lfloor 1 / q h\rfloor, 0<h<1$, nous avons par $(4)$

$$
\begin{aligned}
\frac{F(\vartheta+h)-F(\vartheta)}{h} & =f_{N}(\vartheta)+\frac{1}{h} \int_{0}^{h} \sum_{n \leqslant N} \frac{B(n \vartheta+n t)-B(n \vartheta)}{n} \mathrm{~d} t+O(1) \\
& =f_{N}(\vartheta)+\frac{1}{2} h N-\sum_{\substack{n \leqslant N \\
n \equiv 0(\bmod q)}} \frac{1}{2 n}+O(1) .
\end{aligned}
$$

Comme la dernière somme n'est pas bornée, cela implique bien la conclusion requise.

Lorsque $\vartheta \in \mathbb{R} \backslash(E \cup \mathbb{Q})$, il suffit de considérer des valeurs particulières de $h$ dans (4). Nous choisissons $h:=\mp 1 / 3 q_{m+1}^{2}$ selon que l'indice $m$ vérifie $p_{m} / q_{m}<\vartheta$ ou $p_{m} / q_{m}>\vartheta$. En supposant, pour fixer les idées, que la seconde éventualité a lieu, nous avons alors $B(n t)-B(n \vartheta)=n(t-\vartheta)$ pour tous les entiers $n<q_{m+1}$ dès que $\vartheta<t<\vartheta+h$. La majoration (5) peut donc être remplacée par $\ll x^{2} / q_{m+1}^{2}$ pour $x<q_{m+1}$. Les calculs précédents peuvent alors être reconduits mutatis mutandis avec toujours $N:=\mid 1 / h\rfloor=1 / h$, mais en choisissant cette fois $j=m$ dans (6) et $q=q_{m+1}$ dans (8). Nous obtenons à nouveau (11) pour ces 
valeurs de $h$. Or, en exploitant le lemme 11.3 de [3] comme dans la fin de la démonstration de la Proposition 11.1 de [3], nous avons également

$$
f_{N}(\vartheta)=\sum_{n \leqslant N} \frac{B(n \vartheta)}{n}=\operatorname{sgn}\left(\frac{p_{1}}{q_{1}}-\vartheta\right) \sum_{j \leqslant m}(-1)^{j} \frac{\log q_{j+1}}{2 q_{j}}+c(\vartheta)+o(1) \quad(m \rightarrow \infty),
$$

où $c(\vartheta)$ est une constante ne dépendant que de $\vartheta$. Comme la série (3) diverge, il s'ensuit, en faisant tendre $m$ vers l'infini par valeurs paires ou impaires, que $F$ n'est pas dérivable en $\vartheta$.

Remarque. Une légère modification de la démonstration précédente fournit le renseignement supplémentaire que tout point de $E$ est en fait un point de Lebesgue de $f$. Compte tenu de (11) et de la convergence de la série (2) en $\vartheta \in E$, il suffit en effet de montrer que, lorsque $h$ et $N$ sont définis par (9), nous avons

$$
\frac{1}{ \pm h} \int_{\vartheta}^{\vartheta \pm h}\left|\sum_{n>N} \frac{B(n t)}{n}\right| \mathrm{d} t=o(1) \quad(m \rightarrow \infty) .
$$

Or, nous avons d'une part, grâce à (8)

$$
\frac{1}{ \pm h} \int_{\vartheta}^{\vartheta \pm h}\left|\sum_{N<n \leqslant N^{2}} \frac{B(n t)}{n}\right| \mathrm{d} t \ll \frac{\log N}{q_{m}} \ll \frac{\log q_{m+1}}{q_{m}}=o(1),
$$

et, d'autre part,

$$
\begin{aligned}
\frac{1}{ \pm h} \int_{\vartheta}^{\vartheta \pm h}\left|\sum_{n>N^{2}} \frac{B(n t)}{n}\right|^{2} \mathrm{~d} t & \leqslant \frac{1}{|h|} \int_{0}^{1}\left|\sum_{n>N^{2}} \frac{B(n t)}{n}\right|^{2} \mathrm{~d} t=\sum_{\ell, n>N^{2}} \frac{(\ell, n)}{12|h| \ell n[\ell, n]} \\
& \ll \sum_{d \geqslant 1} \frac{1}{|h| d^{2}}\left(\sum_{n>N^{2} / d} \frac{1}{n^{2}}\right)^{2} \ll \frac{1}{|h| N^{2}} \ll h .
\end{aligned}
$$

Cela implique bien (13) et établit ainsi la propriété indiquée.

Remerciements. Les auteurs prennent plaisir à remercier Michel Balazard et Bruno Martin pour leurs pertinentes remarques sur une version liminaire de ce travail.

\section{Bibliographie}

[1] L. Báez-Duarte, M. Balazard, B. Landreau \& E. Saias, Étude de l'autocorrélation multiplicative de la fonction «partie fractionnaire 》, Ramanujan J. 9 (2005), 215-240.

[2] M. Balazard \& B. Martin, Sur l'autocorrélation multiplicative de la fonction 《partie fractionnaire 》 et une fonction définie par J. R. Wilton, prépublication, arXiv :1305.4395v1.

[3] R. de la Bretèche \& G. Tenenbaum, Séries trigonométriques à coefficients arithmétiques, J. Anal. Math. 92 (2004), 1-79.

[4] M. E. Munroe, Introduction to measure and integration, Addison-Wesley, Reading, Mass., 1953 ; 1959.

[5] J. Rivat \& G. Tenenbaum, Constantes d'Erdős-Turán, Ramanujan J. 9 (2005), 111-121.

[6] W. Rudin, Real and complex analysis, 3ème édition, McGraw-Hill, 1987.

[7] S. Saks, Theory of the integral, 2ème éd. revue et corrigée, Monografie Mat., vol. VII, PWN, Varsovie, 1937 ; réimpression Dover, New York, 1964.

[8] E.C. Titchmarsh, Theory of functions, Oxford University Press, seconde éd., 1939.

[9] D.N. Sarkhel, The pointwise characterization of derivatives of integrals, Proc. Amer. Math. Soc. 63, $\mathrm{n}^{\circ} 1$ (1977), 125-128.

Régis de la Bretèche

Université Paris Diderot-Paris 7

Sorbonne Paris Cité, UMR 7586

Institut de Mathématiques de Jussieu-PRG

Case 7012, F-75013 Paris

regis.delabreteche@imj-prg.fr
Gérald Tenenbaum Institut Élie Cartan

Université de Lorraine

BP 70239

F-54506 Vandœuvre-lès-Nancy Cedex gerald.tenenbaum@univ-lorraine.fr 